\title{
Section introduction \\ Debate: tailored targeted therapy for all - a realistic and worthwhile objective? Introduction to Session 2
}

\author{
lan E Smith
}

The Royal Marsden Hospital and Institute of Cancer Research, Fulham Road, London, SW3 6JJ, UK

Corresponding author: lan E Smith, ian.smith@rmh.nhs.uk

Published: 18 December 2009

This article is online at

http://breast-cancer-research.com/supplements/11/S3/S6
Breast Cancer Research 2009, 11(Suppl 3):S6

(doi:10.1186/bcr2425)

(c) 2009 BioMed Central Ltd
A pre-debate vote showed the audience to be overwhelmingly in favour of the motion.

Dr Per Lønning spoke in favour. He made the point that tailormade therapy should not be confused with implementing simple prognostic or predictive factors that in general do not define direct biological targets but rather should implement biological parameters revealing a variable statistical correlation to outcome. He discussed that breast cancers have recently been separated into five distinct classes based on gene expression profiles: luminal A, luminal B, HER2, basal class, and, finally, so-called normal breast-like tumours. $\mathrm{Dr}$ Lønning argued that the identification of oestrogen receptor as a predictive marker has made endocrine therapy in breast cancer the ultimate targeted or tailored cancer therapy. Over the past decade, tailored therapy with the use of anti-HER2 strategies has revolutionised treatment for around $20 \%$ of cancer patients. The introduction of poly(ADP-ribose) polymerase inhibitors has improved the process of tailoring treatment for patients carrying BRCA1 and BRCA2 defect tumours, and probably also for many additional patients carrying triple-negative tumours. His argument was that the results achieved up to now suggest that tailored therapy for most if not all breast cancer patients in need of systemic treatments is a realistic approach in the near future.

Dr Kathy Pritchard then spoke against the motion. She likewise acknowledged the role of oestrogen receptor and HER2 in targeted therapy, and further discussed gene expression arrays such as OncotypeDX for targeting treatment. She went on to caution against so-called predictive factors for specific chemotherapies including HER2 and topoisomerase $\| \alpha$, demonstrating that their role so far is uncertain given the inconsistency of individual studies addressing the issue. She concluded that neither of these markers were ready for use and that methodologic issues remained, including lack of reproducibility of measurements, publication bias and lack of power of individual studies. She agreed that the motion was laudable in principle but argued that more research was needed.

At the conclusion of the debate, the majority of the audience still supported the motion but a significant minority switched their vote against. Both Dr Lonning and Dr Pritchard could therefore be said to have won.

\section{Competing interests}

The author declares that they have no competing interests.

\section{Acknowledgement}

This article has been published as part of Breast Cancer Research Volume 11 Suppl 3 2009: Controversies in Breast Cancer 2009. The full contents of the supplement are available online at http://breastcancer-research.com/supplements/11/S3. 